\title{
THERMAL ANALYSIS OF LONGITUDINAL A POROUS FIN WITH TEMPERATURE-DEPENDENT INTERNAL HEAT GENERATION USING THE VARIATION OF PARAMETERS METHOD
}

\author{
Osman Güngörr ${ }^{1}$, Cihat Arslantürk ${ }^{2}$ \\ ${ }^{1}$ Technical Scientific Vocational School, Bayburt University \\ Bayburt, Turkey \\ ${ }^{2}$ Faculty of Engineering, Ataturk University \\ Erzurum, Turkey \\ osmangungor@bayburt.edu.tr,carslan@atauni.edu.tr
}

Received: 14 March 2020; Accepted: 23 June 2020

\begin{abstract}
The present study investigates the thermal performance of longitudinal a porous fin with temperature-dependent internal heat generation. The Darcy model is utilized to obtain the differential form of the governing equation that solves the nonlinear temperature distribution equation using the method of variation of parameters. Although this method is applied to solve both linear and nonlinear differential equations, there exist rare applications of this method to solve nonlinear heat transfer problems. In the present study, we applied the method to estimate the thermal analysis of the porous fin exposed to convection. The heat generation is assumed as a function of temperature. The effects of the convection parameter $N_{c}$, internal heat generation $\varepsilon$, porosity $S_{h}$ and generation number $G$ parameter on the dimensionless temperature distribution are discussed in detail. The accuracy of the variation of parameters method is verified through comparison with homotopy perturbation method and the Matlab bvp4c solver (NUM). The results have disclosed that the variation of parameters method can be used as a very effective and practical approach for further studies of the porous medium.
\end{abstract}

MSC 2010: 34L30, 80M30

Keywords: variation of parameters method, porous fin, heat generation, exact solution

\section{Introduction}

Extended surfaces or fins are frequently used as heat dissipation equipment to improve the rate of heat transfer between a hot surface and its surroundings [1]. Their areas of utilization are not restricted by the usual thermal engineering practices such as air conditioning, cooling, chemical industry and radiator designs. They are also very encouraging in the cooling of an electronic circuit of space vehicles. Given the increasing importance of cost-effective, efficient heat dissipa- 
tion systems, the increasing of the heat transfer rate of the fins has been of pivotal significance, therefore, this area has attracted the attention of many researchers. In this respect, thermal analysis of solid fins with various geometries and thermophysical characteristics has been carried out by many researchers $[2,3]$. On the other hand, augmentation of heat transfer owing to a porous fin has a remarkable area of research particularly lately.

Heat dissipation systems in the porous media has a vital role in numerous engineering applications such as heat exchangers, heating, cooling and solar collectors. Kiwan and Al-Nimr [4] firstly introduced the concept of using porous fin so as to enhance the heat transfer rate from a given surface. The thermal performance of the porous fin was also estimated and compared with that of the conventional solid fin. In their work, the results demonstrated that the value of the porosity parameter considerably increases the heat transfer rate from a porous fin as a result of the enhancement in Rayleigh and Darcy numbers. Kiwan [5] introduced the Darcy's model for three types of the porous fin as the long fin, finite-length fin with insulated tip and finite-length fin with known convective coefficient at the tip. Gorla and Bakier [6] examined the thermal analysis of porous fin with a rectangular profile for convection-radiation heat transfer. It is concluded that the radiation effect on the fin is greater than convection only. Kundu et al. [7] presented a model on the performance and optimum design analysis of porous fin with different section profiles for computing the maximum heat transfer rate. Hatami and Ganji [8] theoretically studied the thermal analyses of a circular convection-radiation porous fin with four different materials and shapes. In their work, the governing equations of a porous fin obtained using Darcy's model were analyzed via fourth order Runge-Kutta method and Least Square Method. They concluded that the porous fin with exponential form and silicon nitride material has the maximum quantity of transferred heat compared to other fins. Cuce and Cuce [9] successfully performed the homotopy perturbation method to evaluate fin efficiency and the effectiveness of the longitudinal rectangular profile porous fin. They also designated the ratio of porous fin to the solid fin heat transfer rate as a function of thermo-geometric fin parameters. Kundu and Das [10] investigated the optimum design of thin fins with longitudinal, annular and pin fin profiles. It was observed that closed-form expressions are also obtained for the optimum profiles of longitudinal and pin fins.

The above literature review for porous fin [4-10] has been conducted for the rectangular and annular profile porous fins. However, it is observed that several attempts dealing with more different fin profiles and geometries than these of porous fins are available in the literature. Moradi et al. [11] carried out an investigation on the heat transfer analysis of triangular convection-radiation porous fin with temperature-dependent thermal conductivity. They also compared the results with those of the Differential Transformation Method and fourth order Runge-Kutta method, and obtained an excellent agreement. Kundu and Lee [12] studied the thermal performance analysis and optimal design of annular step porous fin moving with a uniform velocity. They also evaluated the efficiency of porous fin and influences of the Peclet number on temperature distribution. Bhanja and Kundu 
[13] analytically analyzed the thermal performance of T-shape porous fin under the influence of radiation. They underlined that the enhancement in heat transfer is noticed by selecting a porous medium circumstance in the fin. It is obvious from the literature review that the research has been numerously focused on the steady [14-17] and unsteady [18] thermal analysis of cylindrical composite laminates or segments with different directions.

It is obvious from the literature review that various analytical and numerical methods have been employed so far on thermal analysis of porous fin with several geometries and physical characteristics. However, the variation of the parameters method has not been applied to evaluate the thermal analysis of a porous fin with temperature-dependent internal heat generation. Moreover, this method has not been used in heat transfer problems arising in any porous medium conditions as well as porous fin problems. In this respect, the variation of parameters method is applied to thermal analysis of porous fin with a rectangular profile.

The main aim of the present study is to appraise the thermal performance of longitudinal porous fin with thermal-dependent internal heat generation via the variation of parameters method. Based upon the numerical integrations, the variation of parameters method presents an exact solution for the model considered in this paper. It is noted that because numerical integration may be performed to an arbitrary degree of precision, the solution can be considered exact [19].

The variation of parameters method has been principally applied to solve non-homogeneous, linear differential equations. Moreover, this method allows us to effectively solve many engineering problems [19] that arise from engineering applications with appropriate boundary conditions, since it determines unknown parameters in these problems. It is obvious from the literature that this method can also be used to solve nonlinear differential equations [20,21]. These studies have addressed general mathematical aspects of the obtained solutions. However, it is unequivocal from literature [19] that the method of variation of parameters method has been used to solve nonlinear differential equations that arise in various direct and inverse heat transfer applications. Moreover, this method is a simple and relatively easy to implement technique for solving nonlinear equations of the complicated problems such as absorbing and emitting non-gray planar media [22]. Arslantürk [23-25] investigated the validity of this method to estimate temperature distribution in the solid fins with various geometries and to establish optimal dimensions of a space radiator. Güngör and Arslantürk [26] solved the three-dimensional problem of condensation film on an obliqued rotating disk under the steady-state condition using this method.

\section{Description of problem}

Figure 1 shows a porous fin with rectangular geometry. The dimensions of the porous fin are $L, w$ and $t$ which correspond to the length, the width and the thickness of the fin, respectively. 


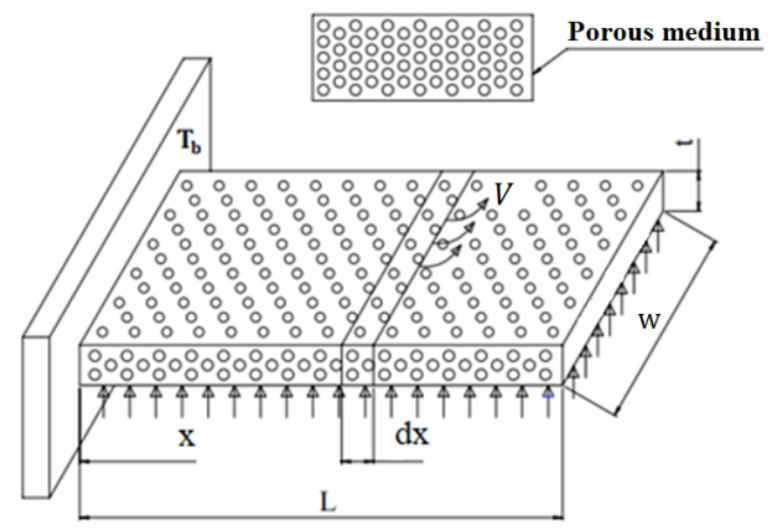

Fig. 1. Schematic diagram of the problem

The cross-section area of the fin is constant, and the fin has a temperature-dependent internal heat generation. The porous medium allows the flow of percolation throughout the fin. Some supposals are accomplished for the governing equations of the proposed model problem. The porous fin is homogenous and isotropic, and it is saturated with single-phase fluid. The radiation effect of the fin surface is regarded to be insignificant. The ambient fluid and fin both have constant properties. The temperature variation across the fin thickness is considered to be negligible. The temperature inside the fin varies only across the fin length. The solid matrix and fluid are assumed to be at local thermal equilibrium with each other. The interactions between the porous medium and the fluid can be simulated by the Darcy formulation.

Based on the above assumptions, the energy balance equation can be expressed as:

$$
q_{x}-q_{x+d x}+q^{*} A d x=\dot{m} c_{p}\left[T(x)-T_{\infty}\right]+h A\left[T(x)-T_{\infty}\right]
$$

where $q$ is the heat flux, $q^{*}$ is the heat generation, $\dot{m}$ is the mass flow rate of the fluid passing through the porous material, $c_{p}$ is the specific heat capacity of the fluid, $h$ is the convective heat transfer coefficient, $A$ is the heat transfer surface area, $T$ is the fin temperature and $T_{\infty}$ is the surrounding temperature.

$V$ is the velocity of fluid passing through the fin at any point that can be determined from Darcy's model [5] as follows:

$$
V=\frac{g K \beta\left(T(x)-T_{\infty}\right)}{v}
$$

where $\mathrm{g}$ is the gravitational acceleration, $K$ is the permeability of the porous fin, $\beta$ is the volumetric thermal expansion coefficient and $v$ is the kinematic viscosity of the fluid. The mass flow rate of the fluid passing through the porous material is:

$$
\dot{m}=\rho V W d x
$$


where $\rho$ is the density of the fluid. The heat transfer surface area is achieved from the fin geometry as:

$$
A=(2 W+2 t) d x
$$

Substitution of Eqs. (2)-(4) into Eq. (1) as $d x \rightarrow 0$ yields:

$$
\frac{d q}{d x}+q^{*} A=\frac{\rho c_{p} g K \beta w}{\mathrm{v}}\left[T(x)-T_{\infty}\right]^{2}+h A\left[T(x)-T_{\infty}\right]
$$

From Fourier's law conduction

$$
q=-k_{e} w t \frac{d T}{d x}
$$

where the effective thermal conductivity of the porous fin $k_{e}$ is relative to both fluid thermal conductivity $k_{f}$ and solid thermal conductivity $k_{s}$ by the following relationship,

$$
k_{e}=\varphi k_{f}+(1-\varphi) k_{s}
$$

where $\varphi$ is the porosity variable. Substitution of Eqs. (6) and (7) into Eq. (5) yields:

$$
\frac{d^{2} T}{d x^{2}}-\frac{\rho c_{p} g K \beta w}{k_{e} t v}\left[T(x)-T_{\infty}\right]^{2}+\frac{h(2 w+2 t)}{k_{e} w t}\left[T(x)-T_{\infty}\right]+\frac{q^{*}}{k_{e}}=0
$$

The heat generation arising inside the porous fin which is variable thermal-dependent can be given by:

$$
q^{*}=q_{\infty}^{*}\left[1+\varepsilon_{a}\left(T-T_{\infty}\right)\right]
$$

where $q_{\infty}^{*}$ is the internal heat generation at temperature $T_{\infty}$. By introducing the following dimensionless parameters

$$
\theta=\frac{T(x)-T_{\infty}}{T_{b}-T_{\infty}} \quad \psi=\frac{x}{L} \quad B i=\frac{h t}{k_{e}} \quad \omega=\frac{L}{t} \quad \varphi=\frac{D a \times R a}{k_{r}}
$$

one gets:

$$
\frac{d^{2} \theta}{d \psi^{2}}-N_{c}^{2} \theta+N_{c}^{2} G(1+\varepsilon \theta)-S_{h} \theta^{2}=0
$$

where $N_{c}$ is the convection parameter, $G$ is the generation number, $\varepsilon$ is the internal heat generation and $S_{h}$ is the porous parameter, which are established as follows:

$$
\begin{gathered}
N_{c}^{2}=2 B i \omega^{2} \quad G=\frac{q_{\infty}^{*}}{2 h\left(T_{b}-T_{\infty}\right)} \\
\varepsilon=\varepsilon_{a}\left(T_{b}-T_{\infty}\right) \quad S_{h}=\varphi \omega^{2}
\end{gathered}
$$


In Eq. (10); $D a, R a$ and $k_{r}$ are the Darcy number, the Rayleigh number and the thermal conductivity ratio, which are given by:

$$
D a=\frac{K}{t^{2}} \quad R a=\frac{\mathrm{g} \beta t^{3}}{v \alpha}\left(T_{b}-T_{\infty}\right) \quad k_{r}=\frac{k_{e}}{k_{f}}
$$

where $K$ and $\alpha$ are the permeability of porous fin and the thermal diffusivity, respectively. In Eq. (11), $S_{h}$ represents the effect of the permeability of the porous medium as well as buoyancy influences. Accordingly, higher permeability of the porous medium attribute to greater values of $S_{h} . N_{c}$ is the convection parameter which indicates the influences of the surface convecting of the fin. On the other hand, $\varepsilon$ is the internal heat generation, and it has vital role in increasing the temperature profile. In this paper, the finite-length fin with insulation is considered. In this respect, because the fin tip is insulated, no heat transfer occurs at the insulated tip, and therefore the boundary conditions are as follows:

$$
\begin{gathered}
\theta(1)=1 \\
\left.\frac{d \theta}{d \psi}\right|_{\psi=0}=0
\end{gathered}
$$

\section{Method of variation of parameters}

Consider a nonlinear differential equation

$$
y^{\prime \prime}(x)+A y^{\prime}(x)+B y(x)=f\left(x, y, y^{\prime}\right)
$$

where $A$ and $B$ are constants and $f$ is a nonlinear function of independent and dependent variable, $x$ and $y$, respectively. The solution scheme of Eq. (17) comprise of the sum of the complementary and particular solutions. The complementary solution is related to the homogeneous equation corresponding to Eq. (17) and is given by

$$
y_{c}(x)=c_{1} y_{1}(x)+c_{2} y_{2}(x)
$$

where $c_{1}$ and $c_{2}$ are constants and $y_{1}$ and $y_{2}$ form a fundamental set of solutions of the homogeneous equation. The particular solution is

$$
y_{p}(x)=v_{1}\left(x, y, y^{\prime}\right) y_{1}(x)+v_{2}\left(x, y, y^{\prime}\right) y_{2}(x)
$$

where:

$$
v_{1}\left(x, y, y^{\prime}\right)=-\int_{0}^{x} \frac{f\left(x^{\prime}, y, y^{\prime}\right) y_{2}\left(x^{\prime}\right)}{W\left(y_{1}, y_{2}\right)} d x^{\prime}
$$




$$
\begin{gathered}
v_{2}\left(x, y, y^{\prime}\right)=\int_{0}^{x} \frac{f\left(x^{\prime}, y, y^{\prime}\right) y_{1}\left(x^{\prime}\right)}{W\left(y_{1}, y_{2}\right)} d x^{\prime} \\
W\left(y_{1}, y_{2}\right)=\left|\begin{array}{ll}
y_{1}, & y_{2} \\
y_{1} & y_{2}^{\prime}
\end{array}\right|=y_{1} y_{2}^{\prime}-y_{1}^{\prime} y_{2}
\end{gathered}
$$

Therefore, the general solution to Eq. (17) can be expressed as follows:

$$
y(x)=c_{1} y_{1}(x)+c_{2} y_{2}(x)+v_{1}\left(x, y, y^{\prime}\right) y_{1}(x)+v_{2}\left(x, y, y^{\prime}\right) y_{2}(x)
$$

The terms $c_{1}$ and $c_{2}$ are established by using the boundary conditions. The functions $v_{1}$ and $v_{2}$ denote functions of a particular solution. Because $y$ exists on both sides Eq. (23), an iterative process is needed to solve for $y$. Here, the solution can still be regarded exact provided that $f$ is only a function of $x$ and $y$. This phenomenon can be attributed to the fact that the integration operations in Eqs. (20) and (21) can be calculated with an arbitrary degree of accuracy. However, if the function $f$ is also a function of the derivatives of $y$, then the solution cannot be considered as an exact solution since these derivatives can also be calculated by the finite difference method [19].

\section{Solution with variation of parameters method}

As the first step of the solution, if Eq. (11) is rearranged with respect to Eq. (17), one gets:

$$
\frac{d^{2} \theta}{d \psi^{2}}=N_{c}^{2} \theta+N_{c}^{2} G(1+\varepsilon \theta)-S_{h} \theta^{2}
$$

The homogeneous equation corresponding to Eq. (24) is separated and integrated twice, yielding complementary solution.

$$
\theta_{c}(\psi)=c_{1} \psi+c_{2}
$$

As expressed in Eq. (19), the particular solution is defined as follows:

$$
\theta_{p}(\psi)=v_{1}(\psi) \psi+v_{2}
$$

The non-dimensional temperature profile is achieved through the sum of the complementary and particular solutions

$$
\theta(\psi)=c_{1} \psi+c_{2}+v_{1}(\psi) \psi+v_{2}(\psi)
$$

In Eq. (25); the constant $c_{1}$ and $c_{2}$ are established by applying the boundary conditions. On the other hand, $v_{1}$ and $v_{2}$ are determined such that it is in the form of Eqs. (20)-(22). Substitution of these unknown parameters into Eq. (27) yields: 


$$
\begin{aligned}
\theta(\psi)=1-\int_{0}^{1} f(\psi, \theta) d \psi & +\int_{0}^{1} \psi f(\psi, \theta) d \psi \\
& +\psi \int_{0}^{\psi} f(\psi, \theta) d \psi-\int_{0}^{\psi} \psi f(\psi, \theta) d \psi
\end{aligned}
$$

Note that $\theta$ denotes a dimensionless temperature profile and appears on both sides of Eq. (28). In that case, an iterative approach is required. An initial guess for the temperature profile is assigned into the right side of this equation. The integrals are evaluated numerically, and a new temperature profile is calculated. This operation is repeated until convergence is achieved [19].

\section{Results and discussion}

This section deals with the analysis of results obtained from variation of parameters method. It is unequivocal that the precision of the variation of parameters approach is restricted only by means of the precision of the numerical integration [19]. As mentioned earlier, the dimensionless temperature profile estimated from Eq. (28) requires an iterative approach. The integrals of this equation are solved numerically via the trapezoidal rule. The accuracy and convergence of the method is supported by comparing the results for $N_{c}=0.3, \varepsilon=0.2, S_{h}=0.1, G=0.4$ to those calculated using the homotopy perturbation method [27] and to the Matlab bvp4c solver (NUM). Figure 2 depicts this comparison and data of it is listed via Table 1. It is observed that there is an excellent agreement between the results of the compared methods.

Table 1. Comparison of the present method, HPM and NUM for various locations

\begin{tabular}{|c|c|c|c|}
\hline$\Psi$ & VPM & HPM [27] & NUM \\
\hline 0.0 & 0.934213428 & 0.934213444 & 0.934213428 \\
\hline 0.1 & 0.934856715 & 0.934856727 & 0.934856715 \\
\hline 0.2 & 0.936788310 & 0.936788323 & 0.936788309 \\
\hline 0.3 & 0.940013429 & 0.940013444 & 0.940013429 \\
\hline 0.4 & 0.944540802 & 0.944540815 & 0.944540802 \\
\hline 0.5 & 0.950382714 & 0.950382725 & 0.950382714 \\
\hline 0.6 & 0.957555080 & 0.957555090 & 0.957555079 \\
\hline 0.7 & 0.966077531 & 0.966077540 & 0.966077531 \\
\hline 0.8 & 0.975973531 & 0.975973539 & 0.975973531 \\
\hline 0.9 & 0.987270505 & 0.987270513 & 0.987270505 \\
\hline 1.0 & 1.000000000 & 1.000000000 & 1.000000000 \\
\hline
\end{tabular}


Figures 3-8 is plotted to show the dimensionless temperature distribution within porous fin variation with porous parameter $S_{h}$, convection parameter $N_{c}$, heat generation parameters $\varepsilon$ and $G$, respectively. Figure 3 illustrates the influence of porosity on the nondimensional temperature profile while remains constant $\left(N_{c}=0.3\right.$, $\varepsilon=0.4, G=0.1)$. It can be clearly understood from Figure 3 that the temperature of porous fin considerably reduces as the values of porous parameter $\left(S_{h}\right)$ increase, therefore, the fin rapidly reaches the ambient temperature. This phenomenon can be explained as a consequence of natural convection. On the other hand, Figure 4 shows the dimensionless temperature profile of porous fin in accordance with convection parameter $\left(N_{c}\right)$. It is observed that the temperature profile decreases with the increasing of the values of parameter $N_{c}$. The reason is that as convection parameter $N_{c}$ increases, the nondimensional temperature at the fin tip remains low.

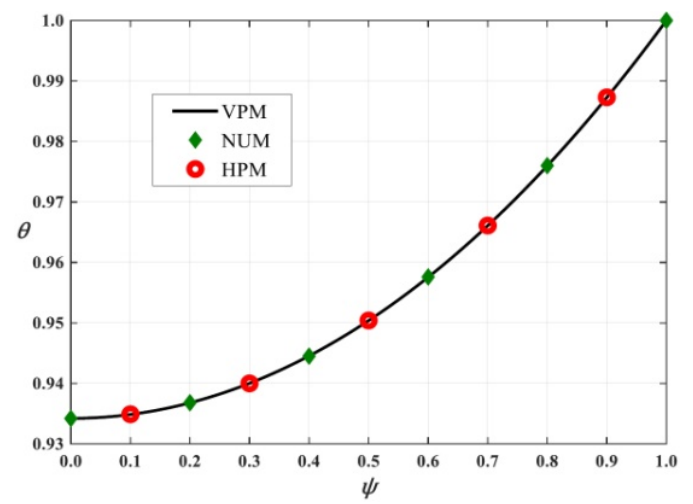

Fig. 2. Comparison of non-dimensional temperature profiles found from VPM to those found from HPM and NUM for $N_{c}=0.3, \varepsilon=0.2, S_{h}=0.1, G=0.4$

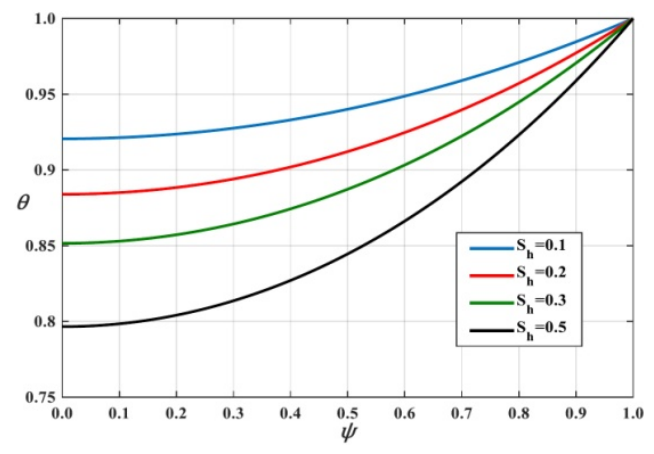

Fig. 3. Non-dimensional temperature distributions for different values of $S_{h}$ when $N_{c}=0.3, \varepsilon=0.4, G=0.1$

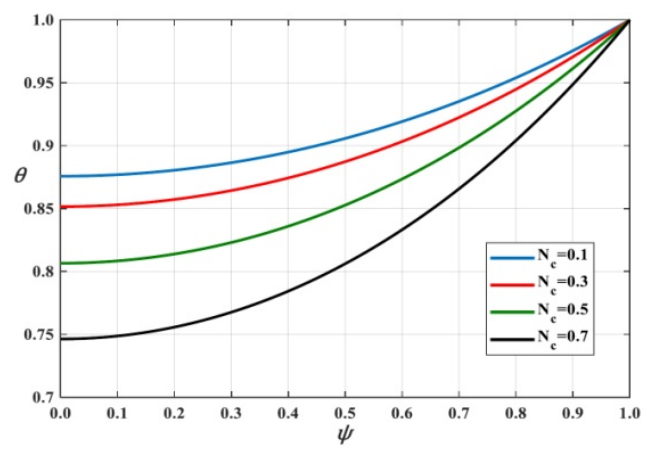

Fig. 4. Non-dimensional temperature distributions for different values of $N_{c}$ when $\varepsilon=0.4, S_{h}=0.3, G=0.1$

The effect of internal heat generation parameter $(\varepsilon)$ on the non-dimensional temperature profile is shown in Figure 5. It can be seen that as the values of the internal heat generation parameter $\varepsilon$ increases, the temperature profile of porous fin 
increases. This finding can attribute to the dissipation of a larger amount of heat to the surroundings under steady state conditions. Similarly, heat generation parameter $(G)$ dependency of the non-dimensional temperature profile is presented in Figure 6.

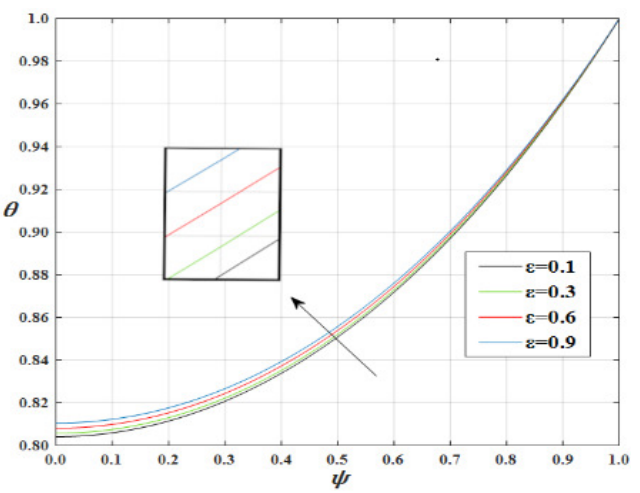

Fig. 5. Non-dimensional temperature distributions for various values of $\varepsilon$ when $N_{c}=0.5, S_{h}=0.3, G=0.1$

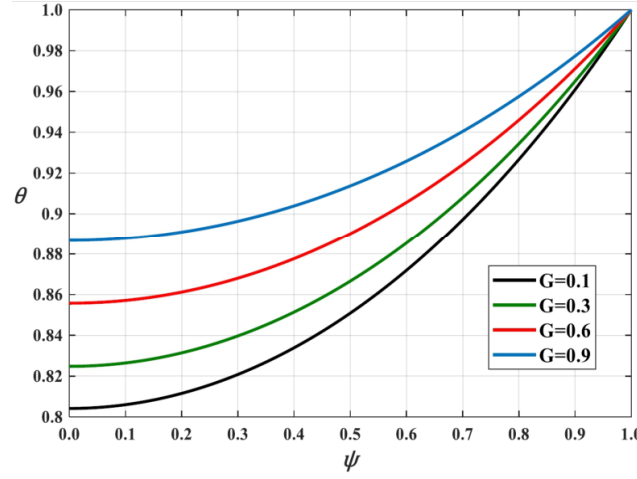

Fig. 6. Non-dimensional temperature distributions for different values of $G$ when $N_{c}=0.5, \varepsilon=0.1, S_{h}=0.3$

Figures 7 and 8 show the effect of heat generation parameters on the tip temperature. It can be stated from these figures that the tip temperature increases with an increase in the values of heat generation parameters. It is also understood that the fin tip temperature changes linearly with the $G$ parameter.

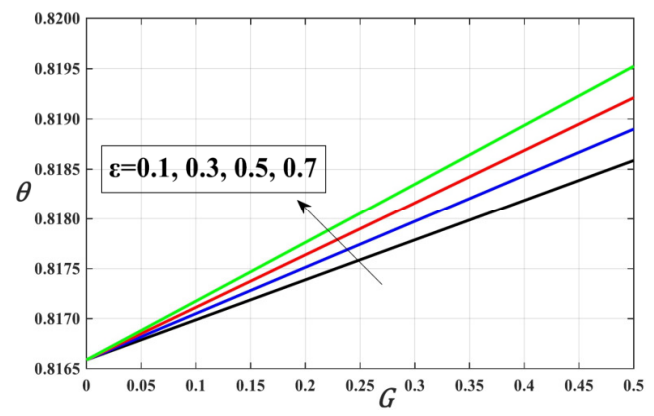

Fig. 7. The effect of heat generation parameters on the tip temperature when $S_{h}=0.5$ and $N_{c}=0.1$

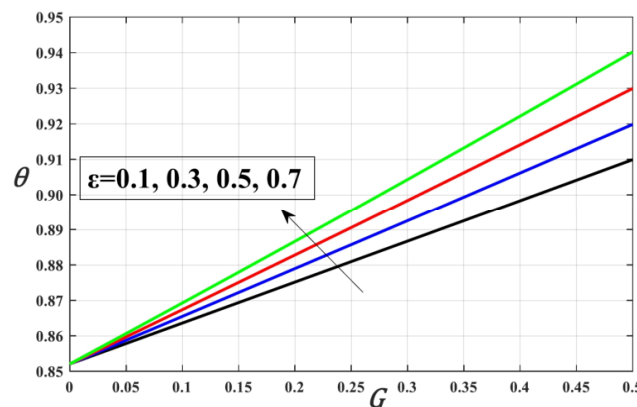

Fig. 8. The effect of heat generation parameters on the tip temperature when $S_{h}=0.1$ and $N_{c}=0.5$

\section{Conclusion}

In this paper, the thermal analysis of the longitudinal porous fin with an internal heat generation parameter with respect to temperature was investigated. The temperature distribution inside the porous fin was calculated via variation of parame- 
ters method, which has recently been successfully applied to nonlinear heat transfer problems. In order to assess the accuracy of the solutions obtained with VPM, this method was compared with HPM and NUM, and it was observed that there was a perfect consistency between the methods. A few physical results of the present study are given as follows:

- The method of variation of parameters is a relatively easy and highly effective approach to applicability compared to other analytical-numerical methods.

- As an expected outcome of natural convection, as the $N_{c}$ value of the convection parameter increases, a reduction in the dimensionless temperature value occurs with the drop in temperature at the fin tip.

- As a result of the increase in the numbers $D a$ and $R a$, it is noted that as the $S_{h}$ value of the porosity parameter increases, the dimensionless temperature profile decreases.

- The variation in heat generation inside the porous fin is directly related to the $\varepsilon$ and $G$ values. As the values of these parameters increase, the dimensionless temperature profile reaches higher values. Under steady state conditions, since the amount of heat dissipated from the porous fin is greater, the dimensionless temperature value is expected to increase further for higher values of heat generation parameters.

\section{References}

[1] Kraus, A.D., Aziz, A., \& Welty, J. (2001). Extended Surface Heat Transfer. New York: John Wiley \& Sons Inc.

[2] Arslanturk, C., (2010). Analysis of thermal performance of annular fins with variable thermal conductivity by homotopy analysis method. Isi Bilimi ve Teknigi Dergisi. Journal of Thermal Science \& Technology, 30(2), 1-7.

[3] Arslanturk C., (2010). Optimization of straight fins with a step change in thickness and variable thermal conductivity by homotopy perturbation method. Isi Bilimi ve Teknigi Dergisi. Journal of Thermal Science \& Technology, 30(2), 9-19.

[4] Kiwan, S., \& Al-Nimr, M.A. (2001). Using porous fins for heat transfer enhancement. Journal of Heat Transfer, 123(4), 790-795.

[5] Kiwan, S. (2007). Effect of radiative losses on the heat transfer from porous fins. International Journal of Thermal Sciences, 46(10), 1046-1055.

[6] Gorla, R.S.R., \& Bakier, A.Y. (2011). Thermal analysis of natural convection and radiation in porous fins. International Communications in Heat and Mass Transfer, 38(5), 638-645.

[7] Kundu, B., Bhanja, D., \& Lee, K.S. (2012). A model on the basis of analytics for computing maximum heat transfer in porous fins. International Journal of Heat and Mass Transfer, 55(25-26), 7611-7622.

[8] Hatami, M., \& Ganji, D.D. (2013). Thermal performance of circular convective-radiative porous fins with different section shapes and materials. Energy Conversion and Management, 76, 185-193.

[9] Cuce, E., \& Cuce, P.M. (2015). A successful application of homotopy perturbation method for efficiency and effectiveness assessment of longitudinal porous fins. Energy Conversion and Management, 93(2015), 92-99. 
[10] Kundu, B., \& Das, P.K. (2005). Optimum profile of thin fins with volumetric heat generation: a unified approach. Journal of Heat Transfer, 127, 945-948.

[11] Moradi, A., Hayat, T., \& Alsaedi, A. (2014). Convection-radiation thermal analysis of triangular porous fins with temperature-dependent thermal conductivity by DTM. Energy Conversion and Management, 77, 70-77.

[12] Kundu, B., \& Lee, K.S. (2016). A proper analytical analysis of annular step porous fins for determining maximum heat transfer. Energy Conversion and Management, 110, 469-480.

[13] Bhanja, D., \& Kundu, B. (2011). Thermal analysis of a constructal T-shaped porous fin with radiation effects. International Journal of Refrigeration, 34(6), 1483-1496.

[14] Kayhani, M.H., Norouzi, M., \& Amiri Delouei, A. (2012). A general analytical solution for heat conduction in cylindrical multilayer composite laminates. International Journal of Thermal Sciences, 52, 73-82.

[15] Delouei, A., Emamian, A., Karimnejad, S., Sajjadi, H., \& Tarokh, A. (2019). On 2D asymmetric heat conduction in functionally graded cylindrical segments: A general exact solution. International Journal of Heat and Mass Transfer, 143, 118515.

[16] Delouei, A., Emamian, A., Karimnejad, S., \& Sajjadi, H. (2019). A closed-form solution for axisymmetric conduction in a finite functionally graded cylinder. International Communications in Heat and Mass Transfer, 108, 104280.

[17] Delouei, A., Emamian, A., Karimnejad, S., Sajjadi, H., \& Jing, D. (2020). Asymmetric conduction in an infinite functionally graded cylinder: Two-dimensional exact analytical solution under general boundary conditions. Journal of Heat Transfer, 142(4), 044505.

[18] Delouei, A., Kayhani, M.H., \& Norouzi, M. (2012). Exact analytical solution of unsteady axi-symmetric conductive heat transfer in cylindrical orthotropic composite laminates. International Journal of Heat and Mass Transfer, 55(15-16), 4427-4436.

[19] Moore, T.J. (2014). Application of Variation of Parameters to Solve Nonlinear Multimode Heat Transfer Problems. Ph. D Thesis, Brigham Young University.

[20] Mohyud-Din, S.T., Noor, M.A., \& Waheed, A. (2010). Variation of parameters method for initial and boundary value problems. World Applied Sciences Journal, 11(5), 622-639.

[21] Rahmatullah., \& Mohyud-Din, S.T. (2013). Variation of parameters method for nonlinear diffusion equations. International Journal of Modern Applied Physics, 3(1), 48-56.

[22] Moore, T.J., \& Jones, M.R. (2014). Analysis of the conduction-radiation problem in absorbing, emitting, non-gray planar media using an exact method. International Journal of Heat and Mass Transfer, 73, 804-809.

[23] Arslantürk, C., (2016). Isıl parametreleri sıcaklıkla değişen iğne kanatların, parametrelerin değişimi yöntemi ile performans analizi. Pamukkale Üniversitesi Mühendislik Bilimleri Dergisi, 22(4), 246-252.

[24] Arslantürk, C., (2018). Variation of parameters method for optimizing annular fins with variable thermal properties. Pamukkale University Journal of Engineering Sciences, 24(1), 1-7.

[25] Arslantürk, C., (2018). Optimization of space radiators accounting for variable thermal conductivity and base-to-fin radiation interaction. Proceedings of the Institution of Mechanical Engineers, Part G: Journal of Aerospace Engineering, 232(1), 121-130.

[26] Güngör, O., \& Arslantürk, C. (2019). Variation of parameters method for a three-dimensional problem of condensation film on an inclined rotating disk. Journal of Applied Mathematics and Computational Mechanics, 18(1), 15-28.

[27] Hoshyar, H.A., Rahimipetroudi, I., Ganji, D.D., \& Majidian, A.R. (2015). Thermal performance of porous fins with temperature-dependent heat generation via the homotopy perturbation method and collocation method. Journal of Applied Mathematics and Computational Mechanics, 14(4), 53-65. 